\title{
SUBMANIFOLDS WITH CONSTANT SCALAR CURVATURE IN A UNIT SPHERE
}

\author{
Xi GuO AND HAIZHONG LI \\ (Received June 27, 2012, revised November 30, 2012)

\begin{abstract}
We study the submanifolds in the unit sphere $\boldsymbol{S}^{n+p}$ with constant scalar curvature and parallel normalized mean curvature vector field. In this case, we can generalize the work of the second author about hypersurfaces in [8] to submanifolds in a unit sphere.
\end{abstract}

1. Introduction and theorems. Let $R^{n+p}(c)$ be an $(n+p)$-dimensional Riemannian manifold with constant sectional curvature c, we also call it a space form. When $c=1$, $R^{n+p}(c)=\boldsymbol{S}^{n+p}$ is the $(n+p)$-dimensional unit sphere space; when $c=0, R^{n+p}(c)=\boldsymbol{E}^{n+p}$ is the $(n+p)$-dimensional Euclidean space. Let $M$ be an $n$-dimensional compact submanifold in $R^{n+p}(c)$, and $e_{1}, \ldots, e_{n}$ a local orthonormal frame of tangent vector fields on $M$, $e_{n+1}, \ldots, e_{n+p}$ a local orthonormal frame of normal vector fields on $M, \omega_{1}, \ldots, \omega_{n}, \omega_{n+1}$, $\ldots, \omega_{n+p}$ its dual coframe field, then the second fundamental form and the mean curvature vector of $M$ are

$$
A=\sum_{i, j, \alpha} h_{i j}^{\alpha} \omega_{i} \otimes \omega_{j} \otimes e_{\alpha}, \quad \mathbf{H}=\sum_{\alpha} H^{\alpha} e_{\alpha}=\frac{1}{n} \sum_{i, \alpha} h_{i i}^{\alpha} e_{\alpha} .
$$

When $p=1$, i.e., $M$ is a hypersurface in $R^{n+1}(c)$, there are many well-known results for hypersurfaces with constant scalar curvature (see [6], [8], [9], [3], [4], [5] ect.).

In [6], Cheng and Yau have proved some well-known results by introducing a self-adjoint differential operator $\square$ on hypersurface defined by

$$
\square f=\sum_{i, j}\left(n H \delta_{i j}-h_{i j}\right) f_{i j},
$$

where $f \in C^{2}(M),\left(f_{i j}\right)$ is its Hessian.

In [8], the second author proved the following rigidity theorems by the study of the above operator $\square$ and some estimates:

THEOREM 1.1 ([8]). Let $M(n \geq 3)$ be an $n$-dimensional compact hypersurface with constant normalized scalar curvature $r$ in the $(n+1)$-dimensional unit sphere $S^{n+1}(1)$. If

(1) $\bar{r} \equiv r-1 \geq 0$,

2000 Mathematics Subject Classification. Primary 53C42; Secondary 53A10.

Key words and phrases. Scalar curvature, mean curvature vector, the second fundamental form.

The research of the authors was supported by NSFC No. 11271214. 
(2) the square norm $|A|^{2}$ of the second fundamental form of M satisfies

$$
n \bar{r} \leq|A|^{2} \leq \frac{n}{(n-2)(n \bar{r}+2)}\left[n(n-1) \bar{r}^{2}+4(n-1) \bar{r}+n\right],
$$

then either $|A|^{2}=n \bar{r}$ and $M$ is totally umbilical, or

$$
|A|^{2}=\frac{n}{(n-2)(n \bar{r}+2)}\left[n(n-1) \bar{r}^{2}+4(n-1) \bar{r}+n\right],
$$

and $M=S^{1}\left(\sqrt{1-a^{2}}\right) \times S^{n-1}(a), a=\sqrt{(n-2) /(n(\bar{r}+1))}$.

THEOREM 1.2 ([8]). Let $M(n \geq 3)$ be an $n$-dimensional compact hypersurface with constant normalized scalar curvature $r$ in the $(n+1)$-dimensional Euclidean space $\boldsymbol{E}^{n+1}$. If the square norm $|A|^{2}$ of the second fundamental form of $M$ satisfies

$$
n r \leq|A|^{2} \leq \frac{n(n-1)}{(n-2)} r,
$$

then $|A|^{2} \equiv n r$ and $M$ is the $n$-dimensional round sphere $S^{n}(a)$ for $a=\sqrt{1 / r}$.

In this paper, we study submanifolds of the unit sphere with constant scalar curvatures and parallel normalized mean curvature vector field, and we discover that above Theorem 1.1 about hypersurfaces still hold for submanifolds. In fact, we prove

THEOREM 1.3. Let $M(n \geq 4)$ be an $n$-dimensional compact submanifold with constant normalized scalar curvature $r$ in the $(n+p)$-dimensional unit sphere $S^{n+p}(1)$. If

(1) $\bar{r} \equiv r-1 \geq 0$,

(2) $\nabla^{\perp} e_{n+1}=0$, where $e_{n+1}$ is a unit normal vector field, which is parallel to $\mathbf{H}$,

(3) the square norm $|A|^{2}$ of the second fundamental form of $M$ satisfies

$$
n \bar{r} \leq|A|^{2} \leq \frac{n}{(n-2)(n \bar{r}+2)}\left[n(n-1) \bar{r}^{2}+4(n-1) \bar{r}+n\right],
$$

then either $|A|^{2}=n \bar{r}$ and $M$ is totally umbilical, or

$$
|A|^{2}=\frac{n}{(n-2)(n \bar{r}+2)}\left[n(n-1) \bar{r}^{2}+4(n-1) \bar{r}+n\right],
$$

and $M=S^{1}\left(\sqrt{1-a^{2}}\right) \times S^{n-1}(a) \hookrightarrow S^{n+1} \hookrightarrow S^{n+p}, a=\sqrt{(n-2) /(n(\bar{r}+1))}$.

REMARK 1.1. Hou and Cheng got some partial results of Theorem 1.3 in [7] and [1], and Cheng also proved a general result on complete submanifolds with constant scalar curvature and parallel normalized mean curvature vector field in Euclidean spaces in [2]. 
2. Preliminaries and lemmas. Let $M$ be an $n$-dimensional compact submanifold in the $(n+p)$-dimensional space form $R^{n+p}(c)$. We shall make use of the following convention on the range of indices:

$$
1 \leq B, C, D \leq n+p, \quad 1 \leq i, j, k \leq n, \quad n+1 \leq \alpha, \beta, \gamma \leq n+p .
$$

We choose a local orthonormal frame field $\left\{e_{1}, \ldots, e_{n}, e_{n+1}, \ldots, e_{n+p}\right\}$ along $M$, where $\left\{e_{i}\right\}_{i=1,2, \ldots, n}$ are tangent to $M$ and $\left\{e_{\alpha}\right\}_{\alpha=n+1, n+2, \ldots, n+p}$ are normal to $M$. Let $\left\{\omega_{B}\right\}$ be the corresponding dual coframe, and $\left\{\omega_{B C}\right\}$ the connection 1-forms on $R^{n+p}(c)$. With restricting on $M$, the second fundamental form, the curvature tensor and the normal curvature tensor can be given by

$$
\begin{gathered}
\omega_{i \alpha}=\sum_{j} h_{i j}^{\alpha} \omega_{j}, \quad A=\sum_{i, j, \alpha} h_{i j}^{\alpha} \omega_{i} \otimes \omega_{j} \otimes e_{\alpha}, \\
d \omega_{i j}-\sum_{k} \omega_{i k} \wedge \omega_{k j}=-\frac{1}{2} \sum_{k, l} R_{i j k l} \omega_{k} \wedge \omega_{l}, \\
d \omega_{\alpha \beta}-\sum_{\gamma} \omega_{\alpha \gamma} \wedge \omega_{\gamma \alpha}=-\frac{1}{2} \sum_{k, l} R_{\alpha \beta k l}^{\perp} \omega_{k} \wedge \omega_{l},
\end{gathered}
$$

and the mean curvature by $\mathbf{H}=\sum_{\alpha} H^{\alpha} e_{\alpha}$, where $H^{\alpha}=\frac{1}{n} \sum_{i} h_{i i}^{\alpha}$.

The covariant derivatives of the second fundamental form are given by

$$
\begin{aligned}
\sum_{k} h_{i j, k}^{\alpha} \omega_{k}= & d h_{i j}^{\alpha}+\sum_{k} h_{k i}^{\alpha} \omega_{k j}+\sum_{k} h_{k j}^{\alpha} \omega_{k i}+\sum_{\beta} h_{i j}^{\beta} \omega_{\beta \alpha} \\
\sum_{l} h_{i j, k l}^{\alpha} \omega_{l}= & d h_{i j, k}^{\alpha}+\sum_{l} h_{l j, k}^{\alpha} \omega_{l i}+\sum_{l} h_{i j, l}^{\alpha} \omega_{l k}+\sum_{l} h_{i l, k}^{\alpha} \omega_{l j} \\
& +\sum_{\beta} h_{i j, k}^{\beta} \omega_{\beta \alpha} .
\end{aligned}
$$

We can define trace-free linear maps $\phi_{\alpha}: T_{X} M \rightarrow T_{X} M$ by

$$
\left\langle\phi^{\alpha} X, Y\right\rangle=\left\langle A^{\alpha} X, Y\right\rangle-H^{\alpha}\langle X, Y\rangle,
$$

where $x \in M, A^{\alpha}$ is the shape operator of $e_{\alpha}, A^{\alpha}\left(e_{i}\right)=-\sum_{j}\left\langle\bar{\nabla}_{e_{i}} e_{\alpha}, e_{j}\right\rangle e_{j}=\sum_{j} h_{i j}^{\alpha} e_{j}$, and $\phi$ is a bilinear map $\phi: T_{x} M \times T_{x} M \rightarrow T_{x}^{\perp} M$ defined by

$$
\phi(X, Y)=\sum_{\alpha=n+1}^{n+p}\left\langle\phi^{\alpha} X, Y\right\rangle e_{\alpha} .
$$

It's easy to check that $|\phi|^{2}=|A|^{2}-n H^{2}$, where $H^{2}=\sum_{\alpha}\left(H^{\alpha}\right)^{2}$.

The Gauss equation is

$$
R_{i j k l}=c\left(\delta_{i k} \delta_{j l}-\delta_{i l} \delta_{j k}\right)+\sum_{\alpha}\left(h_{i k}^{\alpha} h_{j l}^{\alpha}-h_{i l}^{\alpha} h_{j k}^{\alpha}\right) .
$$


In particular

$$
n(n-1)(r-c)=n^{2} H^{2}-|A|^{2},
$$

where $R=n(n-1) r$ is the scalar curvature of $M$. We call $r$ the normalized scalar curvature (see [8], [9], [10]).

The Codazzi equation and the Ricci equation are (see [10])

$$
\begin{gathered}
h_{i j, k}^{\alpha}=h_{i k, j}^{\alpha}, \\
R_{\alpha \beta i j}^{\perp}=\sum_{k}\left(h_{i k}^{\alpha} h_{k j}^{\beta}-h_{j k}^{\alpha} h_{k i}^{\beta}\right) .
\end{gathered}
$$

If the normalized scalar curvature $r$ is a constant with $r \geq c$, then $n^{2} H^{2}-|A|^{2} \geq 0$. We can choose a unit normal vector field $e_{n+1}$ which is parallel to $\mathbf{H}$. Hence we have (see [10])

$$
\begin{gathered}
H^{n+1}=H, \quad H^{\alpha}=0 \quad(n+2 \leq \alpha \leq n+p), \\
\phi_{i j}^{n+1}=h_{i j}^{n+1}-H \delta_{i j}, \quad \phi_{i j}^{\alpha}=h_{i j}^{\alpha}, \quad n+2 \leq \alpha \leq n+p .
\end{gathered}
$$

We introduce a differential operator $\square$ by defining

$$
\square f=\sum_{i, j=1}^{n}\left(n H \delta_{i j}-h_{i j}^{n+1}\right) f_{i j},
$$

where $f \in C^{2}(M),\left(f_{i j}\right)$ is its Hessian.

By use of Codazzi equation, we easily know that the operator $\square$ is self-adjoint. That is, we have

$$
\int_{M} \square f d v=0, \quad f \in C^{2}(M) .
$$

The following lemma was proved in [8] and [9] for hypersurfaces in a space form. In fact, it's still true for submanifolds in a space form.

LEMMA 2.1. Assume that the normalized scalar curvature $r$ is a constant and $r \geq c$, then

$$
|\nabla A|^{2} \geq n^{2}|\nabla H|^{2}
$$

ProOF. From the Gauss equation (2.8), we get $n^{4}\left|\nabla\left(H^{2}\right)\right|^{2}=\left|\nabla\left(|A|^{2}\right)\right|^{2}$ and

$$
\left|\nabla\left(|A|^{2}\right)\right|^{2}=4 \sum_{k}\left(\sum_{i, j, \alpha} h_{i j}^{\alpha} h_{i j, k}^{\alpha}\right)^{2} \leq 4\left(\sum_{i, j, \alpha}\left(h_{i j}^{\alpha}\right)^{2}\right)\left(\sum_{i, j, k, \alpha}\left(h_{i j, k}^{\alpha}\right)^{2}\right)=4|A|^{2}|\nabla A|^{2} .
$$

Hence

$$
4 n^{2} H^{2}\left(n^{2}|\nabla H|^{2}\right)=n^{4}\left|\nabla\left(H^{2}\right)\right|^{2}=\left|\nabla\left(|A|^{2}\right)\right|^{2} \leq 4|A|^{2}|\nabla A|^{2} .
$$

If $r \geq c$, then $n^{2} H^{2} \geq|A|^{2}$, we get (2.15). 
We need the following inequalities in the proof of our Theorem 1.3:

LEMMA 2.2 ([12]). Let $B: R^{n} \rightarrow R^{n}$ be a symmetric linear map such that $\operatorname{tr} B=0$, then

$$
-\frac{n-2}{\sqrt{n(n-1)}}|B|^{3} \leq \operatorname{tr} B^{3} \leq \frac{n-2}{\sqrt{n(n-1)}}|B|^{3},
$$

where $|B|^{2}=\operatorname{tr} B^{2}$, and the equality holds if and only if at least $(n-1)$ eigenvalues of $B$ are equal.

Lemma 2.3 ([13]). Let $C, B: R^{n} \rightarrow R^{n}$ be symmetric linear maps such that $[C, B]=0$ and $\operatorname{tr} C=\operatorname{tr} B=0$, then

$$
-\frac{n-2}{\sqrt{n(n-1)}}|C|^{2}|B| \leq \operatorname{tr}\left(C^{2} B\right) \leq \frac{n-2}{\sqrt{n(n-1)}}|C|^{2}|B| .
$$

LEMMA 2.4 ([11]). Let $B^{1}, B^{2}, \ldots, B^{m}$ be symmetric $(n \times n)$-matrices. Set $S_{\alpha \beta}=$ $\operatorname{tr}\left(B^{\alpha} B^{\beta}\right), S_{\alpha}=S_{\alpha \alpha}, S=\sum_{\alpha} S_{\alpha}$, then

$$
\sum_{\alpha, \beta}\left|B^{\alpha} B^{\beta}-B^{\beta} B^{\alpha}\right|^{2}+\sum_{\alpha, \beta} S_{\alpha \beta}^{2} \leq \frac{3}{2}\left(\sum_{\alpha} S_{\alpha}\right)^{2},
$$

where $|B|^{2}=\operatorname{tr} B^{t} B$.

In [10], the second author calculated the Laplacian of $|\phi|^{2}$ for submanifolds in a unit sphere (also see [14], [15]):

LEMMA 2.5 ([10]). In the same notations as above, we have

$$
\begin{aligned}
\frac{1}{2} \triangle\left(|A|^{2}\right)= & |\nabla A|^{2}+\sum_{i, j, \alpha} n H_{, i j}^{\alpha} h_{i j}^{\alpha}+n|\phi|^{2}+\sum_{i, j, k, \alpha, \beta} n H^{\alpha} h_{i j}^{\alpha} h_{j k}^{\beta} h_{k i}^{\beta} \\
& -\sum_{i, j, k, l}\left(\sum_{\alpha} h_{i j}^{\alpha} h_{k l}^{\alpha}\right)^{2}-\sum_{\alpha, \beta, i, j}\left(R_{\alpha \beta i j}^{\perp}\right)^{2} .
\end{aligned}
$$

3. Proof of Theorem 1.3. Let $\bar{r}=r-1$. Then, for $n^{2} H^{2}-|A|^{2}=n(n-1) \bar{r}$, we have

$$
n^{2} \triangle\left(H^{2}\right)=2 n^{2} H \triangle H+2 n^{2}|\nabla H|^{2}=\triangle|A|^{2} .
$$

If $\nabla^{\perp} e_{n+1}=0$, then $H_{, i}^{\alpha}=H_{, i} \delta_{\alpha n+1}$ and $H_{, i j}^{\alpha}=H_{, i j} \delta_{\alpha n+1}$. From Lemma 2.5, we get

$$
\begin{aligned}
\square(n H)= & \left(n H \delta_{i j}-h_{i j}\right)(n H)_{, i j}=n^{2} H \triangle H-n h_{i j} H_{, i j} \\
= & \frac{1}{2} \triangle\left(|A|^{2}\right)-n^{2}|\nabla H|^{2}-n h_{i j} H_{, i j} \\
= & \left(|\nabla A|^{2}-n^{2}|\nabla H|^{2}\right)+n|\phi|^{2}+\sum_{i, j, k, \alpha, \beta} n H^{\alpha} h_{i j}^{\alpha} h_{j k}^{\beta} h_{k i}^{\beta} \\
& -\sum_{i, j, k, l}\left(\sum_{\alpha} h_{i j}^{\alpha} h_{k l}^{\alpha}\right)^{2}-\sum_{\alpha, \beta, i, j}\left(R_{\alpha \beta i j}^{\perp}\right)^{2} .
\end{aligned}
$$


By use of Lemmas 2.2, 2.3 and (2.12), we have

$$
\begin{aligned}
\sum_{i, j, k, \alpha, \beta} & H^{\alpha} h_{i j}^{\alpha} h_{j k}^{\beta} h_{k i}^{\beta}=\sum_{i, j, k, \beta} H h_{i j}^{n+1} h_{j k}^{\beta} h_{k i}^{\beta} \\
= & \sum_{i, j, k} H h_{i j}^{n+1} h_{j k}^{n+1} h_{k i}^{n+1}+\sum_{\beta=n+2}^{n+p} \sum_{i, j, k} H h_{i j}^{n+1} \phi_{j k}^{\beta} \phi_{k i}^{\beta} \\
= & H \cdot \operatorname{tr}\left(\phi^{n+1}+H I\right)^{3}+\sum_{\beta=n+2}^{n+p} H \phi_{i j}^{n+1} \phi_{j k}^{\beta} \phi_{k i}^{\beta}+\sum_{\beta=n+2}^{n+p} H^{2}\left|\phi^{\beta}\right|^{2} \\
= & H \cdot \operatorname{tr}\left(\phi^{n+1}\right)^{3}+3 H^{2}\left|\phi^{n+1}\right|^{2}+n H^{4}+\sum_{\beta=n+2}^{n+p} H^{2}\left|\phi^{\beta}\right|^{2} \\
& +\sum_{\beta=n+2}^{n+p} \sum_{i, j, k} H \phi_{i j}^{n+1} \phi_{j k}^{\beta} \phi_{k i}^{\beta} \\
\geq & -\frac{n-2}{\sqrt{n(n-1)}}|H| \cdot\left|\phi^{n+1}\right|^{3}+2 H^{2}\left|\phi^{n+1}\right|^{2}+H^{2}|\phi|^{2}+n H^{4} \\
& -\frac{n-2}{\sqrt{n(n-1)}} \sum_{\beta=n+2}^{n+p}|H| \cdot\left|\phi^{n+1}\right|\left|\phi^{\beta}\right|^{2} \\
= & 2 H^{2}\left|\phi^{n+1}\right|^{2}+H^{2}|\phi|^{2}+n H^{4}-\frac{n-2}{\sqrt{n(n-1)}}|H| \cdot\left|\phi^{n+1}\right||\phi|^{2} .
\end{aligned}
$$

Using the Lemma 2.4, we get

$$
\begin{aligned}
\sum_{i, j, k, l} & \left(\sum_{\alpha} h_{i j}^{\alpha} h_{k l}^{\alpha}\right)^{2}+\sum_{\alpha, \beta, i, j}\left(R_{\alpha \beta i j}^{\perp}\right)^{2} \\
= & \sum_{\alpha, \beta}\left[\operatorname{tr}\left(A^{\alpha} A^{\beta}\right)\right]^{2}+\sum_{\alpha \neq n+1, \beta \neq n+1, i, j}\left(R_{\alpha \beta i j}^{\perp}\right)^{2} \\
\leq & {\left[\operatorname{tr}\left(A^{n+1} A^{n+1}\right)\right]^{2}+2 \sum_{\beta \neq n+1}\left(\operatorname{tr} A^{n+1} A^{\beta}\right)^{2}+\frac{3}{2}\left[\sum_{\beta \neq n+1}\left|\phi^{\beta}\right|^{2}\right]^{2} } \\
= & \left|\phi^{n+1}\right|^{4}+2 n H^{2}\left|\phi^{n+1}\right|^{2}+n^{2} H^{4}+2 \sum_{\beta \neq n+1}\left(\operatorname{tr} \phi^{n+1} \phi^{\beta}\right)^{2} \\
& +\frac{3}{2}\left(|\phi|^{2}-\left|\phi^{n+1}\right|^{2}\right)^{2} \\
\leq & \frac{5}{2}\left|\phi^{n+1}\right|^{4}+2 n H^{2}\left|\phi^{n+1}\right|^{2}+n^{2} H^{4}+2\left|\phi^{n+1}\right|^{2}\left(|\phi|^{2}-\left|\phi^{n+1}\right|^{2}\right) \\
& +\frac{3}{2}|\phi|^{4}-3|\phi|^{2}\left|\phi^{n+1}\right|^{2} \\
= & \frac{1}{2}\left|\phi^{n+1}\right|^{4}+2 n H^{2}\left|\phi^{n+1}\right|^{2}+n^{2} H^{4}-|\phi|^{2}\left|\phi^{n+1}\right|^{2}+\frac{3}{2}|\phi|^{4} .
\end{aligned}
$$


From Lemma 2.1, $|\nabla A|^{2} \geq n^{2}|\nabla H|^{2}$, and we have, by putting (3.2) and (3.3) into (3.1),

$\square(n H)$

$$
\begin{aligned}
\geq & n|\phi|^{2}-\frac{n(n-2)}{\sqrt{n(n-1)}}|H| \cdot\left|\phi^{n+1}\right||\phi|^{2}+n H^{2}|\phi|^{2} \\
& -\frac{1}{2}\left|\phi^{n+1}\right|^{4}+|\phi|^{2}\left|\phi^{n+1}\right|^{2}-\frac{3}{2}|\phi|^{4} \\
= & |\phi|^{2}\left[n-\frac{n(n-2)}{\sqrt{n(n-1)}}|H| \cdot|\phi|+n H^{2}-|\phi|^{2}\right] \\
& +\left(|\phi|-\left|\phi^{n+1}\right|\right)\left[\frac{n(n-2)}{\sqrt{n(n-1)}}|H| \cdot|\phi|^{2}-\frac{1}{2}\left(|\phi|-\left|\phi^{n+1}\right|\right)\left(|\phi|+\left|\phi^{n+1}\right|\right)^{2}\right] .
\end{aligned}
$$

We will show the following key claim:

$$
\left(|\phi|-\left|\phi^{n+1}\right|\right)\left(|\phi|+\left|\phi^{n+1}\right|\right)^{2} \leq \frac{32}{27}|\phi|^{3} .
$$

(1) If $|\phi|=0$, then $\left|\phi^{n+1}\right|=0$, and (3.5) holds.

(2) If $|\phi| \neq 0$, define $x=\left|\phi^{n+1}\right| /|\phi|$, so $x \in[0,1]$. We have by a direct calculation

$$
\max _{x \in[0,1]}(1-x)(1+x)^{2}=\frac{32}{27},
$$

so (3.5) still holds, and we complete the proof of the claim.

$$
\text { As } n^{2} H^{2} \geq|A|^{2}=|\phi|^{2}+n H^{2}, n(n-1) H^{2} \geq|\phi|^{2} \text {, then }
$$

$$
\frac{n(n-2)}{\sqrt{n(n-1)}}|H| \cdot|\phi|^{2}-\frac{1}{2}\left(|\phi|-\left|\phi^{n+1}\right|\right)\left(|\phi|+\left|\phi^{n+1}\right|\right)^{2} \geq\left(\frac{n-2}{n-1}-\frac{16}{27}\right)|\phi|^{3} .
$$

If $n \geq 4,(n-2) /(n-1)-16 / 27>0$, so we have

$$
\begin{aligned}
\square(n H) \geq & |\phi|^{2}\left[n-\frac{n(n-2)}{\sqrt{n(n-1)}}|H| \cdot|\phi|+n H^{2}-|\phi|^{2}\right] \\
= & |\phi|^{2}\left[|\phi|+\frac{1}{2}(n-2) \sqrt{\frac{n}{n-1}}|H|+\sqrt{n+\frac{n^{3} H^{2}}{4(n-1)}}\right] \\
& \cdot\left[-|\phi|-\frac{1}{2}(n-2) \sqrt{\frac{n}{n-1}}|H|+\sqrt{n+\frac{n^{3} H^{2}}{4(n-1)}}\right] .
\end{aligned}
$$

It is checked directly that our assumption (1.6), i.e.,

$$
|A|^{2} \leq \frac{n}{(n-2)(n \bar{r}+2)}\left[n(n-1) \bar{r}^{2}+4(n-1) \bar{r}+n\right],
$$


is equivalent to

$$
\begin{aligned}
& \frac{(n-2)^{2}}{4(n-1)^{2}}\left[n(n-1) \bar{r}+|A|^{2}\right] \cdot\left[|A|^{2}+n(n-1) \bar{r}+4(n-1)\right] \\
& \leq\left[n+\frac{n^{2}}{2} \bar{r}-\frac{n-2}{2(n-1)}|A|^{2}\right]^{2} .
\end{aligned}
$$

Noting (3.9) implies $n+\left(n^{2} / 2\right) \bar{r}-(n-2)|A|^{2} /(2(n-1))>0$, we know (3.10) is equivalent to

$$
\begin{aligned}
& \frac{n-2}{2(n-1)} \sqrt{\left[n(n-1) \bar{r}+|A|^{2}\right] \cdot\left[|A|^{2}+n(n-1) \bar{r}+4(n-1)\right]} \\
& \quad \leq n+\frac{n^{2}}{2} \bar{r}-\frac{n-2}{2(n-1)}|A|^{2} .
\end{aligned}
$$

We note that (3.11) is equivalent to

$$
|\phi| \leq-\frac{1}{2}(n-2) \sqrt{\frac{n}{n-1}}|H|+\sqrt{n+\frac{n^{3} H^{2}}{4(n-1)}} .
$$

Therefore, the right-hand side of (3.8) is non-negative. From (2.14) we also have $\int_{M} \square(n H) d v=0$.

Thus either $|A|^{2}=n \bar{r}$, that is $|\phi|=0$ or

$$
|A|^{2}=\frac{n}{(n-2)(n \bar{r}+2)}\left[n(n-1) \bar{r}^{2}+4(n-1) \bar{r}+n\right],
$$

and if (3.13) holds, we get $|\phi|=\left|\phi^{n+1}\right|$.

Let $N_{1}$ be the sub-bundle spanned by $\left\{e_{n+2}, \ldots, e_{n+p}\right\}$. Then, with the assumption $\nabla^{\perp} e_{n+1}=0$, it's easy to check that $N_{1}$ is parallel in the normal bundle. If $|\phi|=0$ or $|\phi|=\left|\phi^{n+1}\right|$, we can always get that $\left|\phi^{\alpha}\right|=0$ for each $n+2 \leq \alpha \leq n+p$, that means $M$ is totally geodesic with respect to $N_{1}$. So from [15, Theorem 1] we know that $M$ lies in $S^{n+1}$, which is an $(n+1)$-dimensional totally geodesic submanifold in $S^{n+p}$. Using Theorem 1.1, we complete the proof of Theorem 1.3.

REMARK 3.1. If $n=3$, we have from the first inequality of (3.4)

$$
\begin{aligned}
\square(n H) & \geq|\phi|^{2}\left[3+3 H^{2}-\frac{3}{2}|\phi|^{2}-\frac{3}{\sqrt{6}}|H| \cdot\left|\phi^{n+1}\right|+\frac{1}{2}\left|\phi^{n+1}\right|^{2}\right] \\
& =|\phi|^{2}\left[3+\frac{9}{4} H^{2}-\frac{3}{2}|\phi|^{2}+\frac{1}{2}\left(\left|\phi^{n+1}\right|-\sqrt{\frac{3}{2}}|H|\right)^{2}\right] \\
& \geq|\phi|^{2}\left[3+\frac{9}{4} H^{2}-\frac{3}{2}|\phi|^{2}\right] .
\end{aligned}
$$

Hence if $|\phi|^{2} \leq 2+\frac{3}{2} H^{2}$, which is equivalent to

$$
|A|^{2} \leq 4+6 \bar{r}
$$


we get that $M$ is totally umbilical. We also note that (1.6) is equivalent to

$$
n \bar{r} \leq|A|^{2} \leq \frac{n(n-1)}{n-2} \bar{r}+\frac{2(n-1)}{n-2}+\frac{n-2}{n \bar{r}+2} .
$$

Thus, when $n=3$, (3.16) is

$$
|A|^{2} \leq 4+6 \bar{r}+\frac{1}{3 \bar{r}+2} .
$$

Thus (3.15) is stronger than (3.17).

REMARK 3.2. When $n=3$, our technique here is not effective to prove Theorem 1.3, so it is an interesting problem to know that Theorem 1.3 holds or not for $n=3$.

Acknowledgments. The authors would like to thank the referee for some useful comments.

\section{REFERENCES}

[1] Q.-M. Cheng, Submanifolds with constant scalar curvature, Proc. Royal Soc. Edingburgh 132A (2002), 1163-1183.

[2] Q.-M. CHENG, Topology and geometry of complete submanifolds in Euclidean spaces, Banach center publications 69 (2005), 67-80.

[ 3 ] Q.-M. Cheng, Hypersurfaces in a unit sphere $S^{n+1}$ with constant scalar curvature, J. London Math. Soc. 64 (2003), 49-56.

[4] Q.-M. Cheng, Compact hypersurfaces in a unit sphere with infinite fundamnetal group, Pacific J. Math. 212 (2001), 755-768.

[ 5 ] Q.-M. Cheng, S. Shu And Y. J. SuH, Compact hypersurfaces in a unit sphere, Proceedings of the Royal Society of Edinburgh 135A (2005), 1129-1137.

[6] S.-Y. ChEng AND S.-T. YAU, Hypersurfaces with constant scalar curvature, Math. Ann. 225 (1977), 195204.

[ 7 ] Z.-H. Hou, Submanifolds of constant scalar curvature in a space form, Kyun. Math. J. 38 (1998), 439-458.

[ 8 ] H. Li, Hypersurfaces with constant scalar curvature in space forms, Math. Ann. 305 (1996), 665-672.

[ 9 ] H. LI, Global rigidity theorems of hypersurfaces, Ark. Mat. 35 (1997), 327-351.

[10] H. LI, Willmore submanifolds in a sphere, Math. Res. Letters 9 (2002), 771-790.

[11] A.-M. Li AND J.-M. Li, An intrinsic rigidity theorem for minimal submanifolds in a sphere, Arch. Math. 58 (1992), 582-594.

[12] M. OKumura, Hypersurfaces and a pinching problem on the second fundamental tensor, Amer. J. Math. 96 (1974), 207-213.

[13] W. Santos, Submanifolds with parallel mean curvature vector in spheres, Tohoku Math. J. 46 (1994), $403-$ 415.

[14] J. Simons, Minimal varieties in Riemannian manifolds, Ann. of Math. (2) 88 (1968), 62-105.

[15] S.-T. YAU, Submanifolds with constant mean curvature I, Amer. J. Math. 96 (1974), 346-366.

Department of Mathematical SCiences

TSINGHUA UNIVERSITY

100084, BEIJING

P. R. CHINA

E-mail addresses: x-guo08@mails.tsinghua.edu.cn hli@math.tsinghua.edu.cn 